\title{
Swiss cheeses, rational approximation and universal plane curves
}

\author{
by \\ J. F. Feinstein (Nottingham) and M. J. Heath (Lisboa)
}

\begin{abstract}
We consider the compact plane sets known as Swiss cheese sets, which are a useful source of examples in the theory of uniform algebras and rational approximation. We develop a theory of allocation maps connected to such sets and we use this theory to modify examples previously constructed in the literature to obtain examples homeomorphic to the Sierpiński carpet. Our techniques also allow us to avoid certain technical difficulties in the literature.
\end{abstract}

1. Introduction and motivation. In this paper we shall concern ourselves with "Swiss cheese" constructions. These represent a particular method for constructing compact subsets of the complex plane that has been used extensively in the theory of rational approximation and, more generally, in the theory of uniform algebras. In general, little is specified about the topology of the sets produced by this technique. Since uniform algebra theory has strong connections to topology, the topological properties of the sets on which we build our examples is an obvious thing to study. In this paper we shall show that it is possible to modify many Swiss-cheesebased examples related to uniform algebras and rational approximation in such a way that our compact plane set is homeomorphic to the well-known Sierpiński carpet.

1.1. Basic uniform-algebraic concepts. Throughout this paper by a compact space we will mean a non-empty, compact, Hausdorff topological space. Let $X$ be a non-empty, locally compact, Hausdorff space. We denote by $C_{0}(X)$ the set of all continuous functions from $X$ to $\mathbb{C}$ which tend to zero at infinity. If $X$ is a compact space, this is equal to the set of all continuous $\mathbb{C}$-valued functions, which we denote by $C(X)$. Equipping $C(X)$ with the usual pointwise operations makes it a commutative, semisimple, complex algebra. If we further equip $C(X)$ with the supremum norm $\|\cdot\|_{\infty}$,

2010 Mathematics Subject Classification: Primary 46J10; Secondary 54H99.

Key words and phrases: Swiss cheeses, rational approximation, uniform algebras. 
it is standard that it is then a Banach algebra. We always treat $C(X)$ as a Banach algebra with this norm. We call a closed subalgebra $A$ of $C(X)$ a uniform algebra on $X$ if it contains the constant functions and if, for all $x, y \in X$ with $x \neq y$, there is $f \in A$ with $f(x) \neq f(y)$. A uniform algebra on $X$ is trivial if it is equal to $C(X)$ and non-trivial otherwise.

A character on a uniform algebra $A$ is a non-zero algebra homomorphism from $A$ into $\mathbb{C}$. A uniform algebra $A$ on a compact space $X$ is natural if the only characters from $A$ into $\mathbb{C}$ are evaluations at points of $X$.

We shall use the term plane set to mean "subset of the complex plane". For a non-empty, compact plane set $X$ we define $R_{0}(X)$ to be the subalgebra of $C(X)$ consisting of functions $f=\left.g\right|_{X}$ where $g: \mathbb{C} \rightarrow \mathbb{C} \cup\{\infty\}$ is a rational function with $\infty \notin g(X)$. We define $R(X)$ to be the supremum-norm closure of $R_{0}(X)$ in $C(X)$. It is standard that $R(X)$ is a natural uniform algebra on $X$.

Definition 1.1. A uniform algebra, $A$, on a compact space, $X$, is essential if, for each non-empty, proper, closed subset, $Y$, of $X$, there is a function $f \in C(X) \backslash A$ such that $\left.f\right|_{Y}=0$.

Definition 1.2. Let $X$ be a compact space, let $\mu$ be a regular Borel measure on $X$ and let $U \subset C(X)$. We say that $\mu$ is an annihilating measure for $U$ if $\int_{X} f d \mu=0$ for all $f \in U$. We shall denote by $M(X)$ the Banach space of regular, complex Borel measures on $X$ with the total variation norm.

The following result is [4, Theorem 2.8.1] together with some observations made in the proof of that theorem.

Proposition 1.3. Let $A$ be a uniform algebra on a compact space, $X$, and let $E(A)$ be the closure in $X$ of the union of the supports of all annihilating measures for $A$ on $X$. Then $E(A)$ is the unique, minimal, closed subset of $X$ such that, for all $f \in C(X)$ with $f(E(A)) \subseteq\{0\}$, we have $f \in A$. Furthermore, $\left.A\right|_{E(A)}$ is uniformly closed in $C(E(A))$, and

$$
A=\left\{f \in C(X):\left.\left.f\right|_{E} \in A\right|_{E}\right\} .
$$

The uniform algebra $A$ is essential if and only if $E(A)=X$, and $A=C(X)$ if and only if $E(A)=\emptyset$.

We may think of the essential set of $A$ as being "the set on which $A$ is non-trivial" and "essential" as meaning "everywhere non-trivial".

Definition 1.4. Let $A$ be a commutative Banach algebra and let $\psi$ be a character on $A$. A point derivation at $\psi$ is a linear functional $d$ on $A$ such that

$$
d(a b)=\psi(a) d(b)+\psi(b) d(a) \quad \text { for all } a, b \in A .
$$


Let $n \in \mathbb{N} \cup\{\infty\}$. A point derivation of order $n$ at $\phi$ is a sequence $\left(d_{k}\right)_{k=0}^{n}$ of linear functionals such that $d_{0}=\phi$ and, for each $i<n+1$,

$$
d_{i}(f g)=\sum_{k=0}^{i} d_{k}(f) d_{i-k}(g) .
$$

We call $\left(d_{k}\right)_{k=0}^{n}$ bounded if, for each $i<n+1, d_{i}$ is a bounded linear functional.

Let $A$ be a natural uniform algebra on a compact space $X$. We say that: $A$ is regular if, for all $x \in X$ and all compact sets $E \subseteq X \backslash\{x\}$, there exists $f \in A$ such that $f(E) \subseteq\{1\}$ and $f(x)=0 ; A$ is normal if, for every closed set $F \subseteq X$ and every compact set $E \subseteq X \backslash F$, there exists $f \in A$ such that $f(E) \subseteq\{1\}$ and $f(F) \subseteq\{0\}$. It is standard that regularity and normality are equivalent for natural uniform algebras (see [6, Proposition 4.1.18]).

1.2. Connections between uniform algebras and topology. In order to motivate our results we shall discuss connections between the theory of uniform algebras and topology. The key observation is the following, which is basically trivial.

Proposition 1.5. Let $\mathrm{P}$ be a property, which a Banach algebra may have and which is invariant under Banach algebra isomorphism. Then, for a compact space $X$, "there exists a uniform algebra on $X$ which satisfies $\mathrm{P}$ " and "there exists a natural uniform algebra on $X$ which satisfies $\mathrm{P}$ " are topological properties of $X$.

Thus, for a compact space $X$, it makes sense to consider questions of the form: "Which Banach-algebraic properties may a (natural) uniform algebra on $X$ have?" These sorts of question have been little studied. In the many examples of uniform algebras constructed using Swiss cheese techniques there is not typically any mention made of the topological properties of the underlying compact space.

Now, an obvious technique for constructing uniform algebras with different sets of properties on a fixed compact space is as follows. Let $Y$ be a compact space, let $X$ be a compact subspace of $Y$, and let $A$ be a uniform algebra on $X$. We may define a uniform algebra, $A^{(Y)}$, on $Y$ thus:

$$
A^{(Y)}=\left\{f \in C(Y):\left.f\right|_{X} \in A\right\} .
$$

Many properties of $A$ are then necessarily shared by $A^{(Y)}$. For example the following are easily proven and probably all well-known (see, for example, [13. Lemma 2.4.9] for details).

Lemma 1.6. Let $A$ be a uniform algebra on a compact space $X$, and let $Y$ be a compact space such that $X \subseteq Y$. Then:

(a) $A^{(Y)}$ is trivial if and only if $A$ is trivial; 
(b) $A^{(Y)}$ is natural if and only if $A$ is natural;

(c) $A^{(Y)}$ is normal if and only if $A$ is normal;

(d) if $z \in X, n \in \mathbb{N} \cup\{\infty\}$ and $A$ has a non-zero bounded point derivation of order $n$ at $z$, then $A^{(Y)}$ has a non-zero, bounded point derivation of order $n$ at $z$.

Hence we may, for example, construct a non-trivial, natural, normal, uniform algebra on the compact unit disc. These examples are somewhat artificial, since they are not essential.

1.3. A survey of the use of Swiss cheese constructions in the theory of uniform algebras. Examples in the theory of uniform algebras are often constructed by considering compact subsets of the complex plane obtained by removing some sequence of open discs from a compact disc. Sets built in such a way are usually called "Swiss cheeses" or "Swiss cheese sets"; we shall use the term "Swiss cheese" in a related but different sense, and we note that every compact plane set may be constructed in this way. We let $X$ be a compact plane set constructed by means of a Swiss cheese and consider the uniform algebra $R(X)$. By placing conditions on the radii and centres of the discs to be removed we are able to control certain Banach-algebraic properties of $R(X)$.

For a (closed or open) disc $D$ in the plane, we let $r(D)$ be the radius of $D$. If $D$ is the empty set or a singleton we say $r(D)=0$.

Definition 1.7. We shall call a pair, $\mathbf{D}=(\Delta, \mathcal{D}) \in \mathcal{P}(\mathbb{C}) \times \mathcal{P}(\mathcal{P}(\mathbb{C}))$, a Swiss cheese if $\Delta$ is a compact disc and $\mathcal{D}$ is a countable or finite collection of open discs. Let $\mathbf{D}=(\Delta, \mathcal{D})$ be a Swiss cheese. We say that $\mathbf{D}$ is: semiclassical if the discs in $\mathcal{D}$ intersect neither one another, nor $\mathbb{C} \backslash \Delta$, and if, for each $D \in \mathcal{D}, \bar{D} \subsetneq \Delta$ and $\sum_{D \in \mathcal{D}} r(D)<\infty$; classical if the closures of the discs in $\mathcal{D}$ intersect neither one another nor $\mathbb{C} \backslash$ int $\Delta$, and $\sum_{D \in \mathcal{D}} r(D)<\infty$; finite if $\mathcal{D}$ is finite.

Let $\mathbf{D}=(\Delta, \mathcal{D})$ be a Swiss cheese. We call the plane set $X_{\mathbf{D}}:=\Delta \backslash \bigcup \mathcal{D}$ the associated Swiss cheese set. We say that a plane set $X$ is: a semiclassical Swiss cheese set if there is a semiclassical Swiss cheese $\mathbf{D}$ such that $X=X_{\mathbf{D}}$; a classical Swiss cheese set if there is a classical Swiss cheese $\mathbf{D}$ such that $X=X_{\mathbf{D}}$.

The earliest use of a Swiss cheese set in the theory of rational approximation was in [19], where Roth constructed a classical Swiss cheese set $K$ with empty interior such that $R(K) \neq C(K)$. This showed that there are compact plane sets such that $R(X) \neq A(X)$ where $A(K)$ is the uniform algebra of continuous functions on $K$ which are analytic on the interior of $K$. Roth's proof was essentially the same as that of Theorem 1.8 , below. 
A second example, showing how careful choice of the discs to be removed allows us to control the properties of $R(X)$ was given by Steen in [22]. This example is a classical Swiss cheese set $X$ such that $R(X)$ contains a nonconstant, real-valued function, something that had been conjectured to be impossible. Furthermore, this function depended only on the real part of the independent variable.

We shall concentrate on Swiss cheeses D such that the associated Swiss cheese set, $X_{\mathbf{D}}$, has empty interior in $\mathbb{C}$. We mention in passing that Swiss cheese sets with non-empty interior are used, for example, in Examples 9.19.3 of [11, to demonstrate that a compact plane set $K$ may have dense interior and yet have $R(K) \neq A(K)$.

We introduce some notation for integration over paths and chains. Further details may be found in Chapter 10 of [20]. Let $\left(\gamma_{1}, \ldots, \gamma_{k}\right)$ and $\left(\delta_{1}, \ldots, \delta_{n}\right)$ be finite sequences of piecewise smooth paths in the plane. We say $\left(\gamma_{1}, \ldots, \gamma_{k}\right)$ and $\left(\delta_{1}, \ldots, \delta_{n}\right)$ are equivalent if, for all $f \in C_{0}(\mathbb{C})$, we have

$$
\sum_{i=1}^{k} \int_{\gamma_{i}} f d z=\sum_{i=1}^{n} \int_{\delta_{i}} f d z .
$$

It is standard that this defines an equivalence relation on the set of all such sequences; we call the equivalence classes induced by this relation chains. We denote the chain containing $\left(\gamma_{1}, \ldots, \gamma_{k}\right)$ by $\gamma_{1} \dot{+} \cdots \dot{+} \gamma_{k}$. Let $\Gamma=\gamma_{1} \dot{+} \cdots \dot{+} \gamma_{k}$. We define integration over $\Gamma$ as follows:

$$
\int_{\Gamma} f d z:=\sum_{i=1}^{k} \int_{\gamma_{i}} f d z \quad\left(f \in C_{0}(\mathbb{C})\right) .
$$

If $\gamma_{1}, \ldots, \gamma_{n}$ are chains we write $\gamma_{1} \dot{+} \cdots \dot{+} \gamma_{n}$ for the chain $\Gamma$ with

$$
\int_{\Gamma} f d z=\int_{\gamma_{1}} f d z+\cdots+\int_{\gamma_{n}} f d z \quad\left(f \in C_{0}(\mathbb{C})\right) .
$$

For a chain or piecewise smooth path $\gamma$ we define $\mu_{\gamma}$ to be the unique, regular, Borel measure on $\mathbb{C}$ satisfying

$$
\int_{\mathbb{C}} f d \mu_{\gamma}=\int_{\gamma} f d z \quad\left(\text { for all } f \in C_{0}(\mathbb{C})\right) .
$$

TheOrem 1.8. Let $X$ be a semiclassical Swiss cheese set. Then $R(X)$ is essential.

Proof. Suppose first that $z \in \operatorname{int}(X)$ and let $r>0$ be sufficiently small that $\overline{B(z, \gamma)} \subseteq X$. We define a path $\gamma:[\pi, \pi] \rightarrow \mathbb{C}$ by $\gamma_{z, r}(t)=z+r e^{i t}$. Then, by Cauchy's theorem, $\mu_{\gamma_{z, r}}$ is an annihilating measure for $R(X)$ and so, by Proposition $1.3, z \in E(R(X))$. We shall show that there exists an annihilating measure, $\mu$, for $R(X)$ with $\operatorname{supp}(\mu)=\partial X$ and so, by Proposition 1.3 again, $\partial X \subseteq E(R(X))$. Hence we will have shown that $X=E(R(X))$. 
Let $\mathbf{D}=(\Delta, \mathcal{D})$ be a semiclassical Swiss cheese such that $X=X_{\mathbf{D}}$. We let $\gamma_{\Delta}$ be the boundary circle of $\Delta$ given the positive orientation. For $D \in \mathcal{D}$, let $\gamma_{D}$ be the boundary circle of $D$ given the negative orientation. Obviously $\operatorname{supp}\left(\mu_{\gamma_{\Delta}}\right)=\partial \Delta, \operatorname{supp}\left(\mu_{\gamma_{D}}\right)=\partial D(D \in \mathcal{D})$ and these measures are non-atomic.

Now, for each $D \in \mathcal{D},\left\|\mu_{\gamma_{D}}\right\| \leq 2 \pi r(D)$. Hence

$$
\sum_{D \in \mathcal{D}}\left\|\mu_{\gamma_{D}}\right\| \leq 2 \pi \sum_{D \in \mathcal{D}} r(D)<\infty
$$

so

$$
\mu:=\mu_{\gamma_{\Delta}}+\sum_{D \in \mathcal{D}} \mu_{\gamma_{D}}
$$

defines a measure $\mu \in M(X)$. Clearly, if $Y$ is a closed subset of $\operatorname{int}(X)$ then $\mu(Y)=0$, so $\operatorname{supp}(\mu) \subseteq \partial X$. To show the reverse inclusion, first note that for $D \in \mathcal{D} \cup\{\Delta\}$ and $z \in X, \mu_{\gamma_{D}}(\{z\})=0$. Now let $Y$ be a closed subset of $\partial \Delta$. Then $Y \cap \bigcup_{D \in \mathcal{D}} \bar{D}$ is countable and so $\mu\left(Y \cap \bigcup_{D \in \mathcal{D}} \bar{D}\right)=0$ and $\mu_{\Delta}\left(Y \cap \bigcup_{D \in \mathcal{D}} \bar{D}\right)=0$. Hence $\mu(Y)=\mu_{\Delta}(Y)$. Similarly, if we let $D \in \mathcal{D}$ and $Y \subseteq \partial D$ then $\mu(Y)=\mu_{D}(Y)$. Hence, for each point $z \in \partial \Delta \cup \bigcup_{D \in \mathcal{D}} \partial D$ and every neighbourhood $U$ of $z$ there is a set $Y \subseteq U$ with $\mu(Y) \neq 0$. Thus, $z \in$ $\operatorname{supp}(\mu)$ and so $\partial \Delta \cup \bigcup_{D \in \mathcal{D}} \partial D \subseteq \operatorname{supp}(\mu)$. However, $\overline{\partial \Delta \cup \bigcup_{D \in \mathcal{D}} \partial D}=\partial X$, so we have $\partial X \subseteq \operatorname{supp}(\mu)$. Thus $\partial X=\operatorname{supp}(\mu)$. It only remains to show that $\mu$ is an annihilating measure for $R(X)$.

To show this we let $f \in R_{0}(X)$; then $f$ is holomorphic on the open set

$$
V:=\mathbb{C} \backslash\{z \in \mathbb{C}: z \text { is a pole of } f\} .
$$

We shall assume that $\mathcal{D}$ is infinite; the proof in the case where $\mathcal{D}$ is finite is similar (and easier). We let $\left(D_{n}\right)_{n \in \mathbb{N}}$ be a sequence enumerating $\mathcal{D}$ and pick $N \in \mathbb{N}$ such that those poles of $f$ which are contained in $\Delta$ all lie in $D_{1} \cup \cdots \cup D_{N}$. Then

$$
\Gamma_{N}=\gamma_{\Delta} \dot{+} \gamma_{D_{1}} \dot{+} \cdots \dot{+} \gamma_{D_{N}}
$$

is a cycle with $\operatorname{Ind}(\Gamma, z)=0$ for all $z \in \mathbb{C} \backslash V$. Hence, by Cauchy's theorem,

$$
\int_{\Gamma_{N}} f d z=0,
$$

and letting $N$ tend to infinity yields $\int f d \mu=0$. Hence $\mu$ is an annihilating measure for $R(X)$.

The first known example of a non-trivial uniform algebra with no nonzero bounded point derivations was due to Wermer, [23. In fact, he proved the following. 
Proposition 1.9. Let $\Delta$ be a closed disc in $\mathbb{C}$, and let $\varepsilon>0$. Then there is a classical Swiss cheese $\mathbf{D}=(\Delta, \mathcal{D})$ such that

$$
\sum_{D \in \mathcal{D}} r(D)<\varepsilon,
$$

and $R\left(X_{\mathbf{D}}\right)$ has no non-zero bounded point derivations.

The first known example of a non-trivial, normal uniform algebra was due to McKissick, [16]. In fact, he proved the following.

Proposition 1.10. For any closed disc $\Delta$ and any $\varepsilon>0$, there is a Swiss cheese $\mathbf{D}=(\Delta, \mathcal{D})$ such that

$$
\sum_{D \in \mathcal{D}} r(D)<\varepsilon,
$$

and $R\left(X_{\mathbf{D}}\right)$ is normal.

This construction was simplified somewhat by Körner in [15. In [18] O'Farrell showed that in the above we could further insist that $0 \in X_{\mathbf{D}}$ and that $R(X)$ have a bounded point derivation of infinite order at 0 . The constructions of McKissick, Körner and O'Farrell appeared not to produce classical Swiss cheese sets. McKissick's result, along with what we would now call a system of Cole extensions (see, for example, 7]) was a crucial tool in Cole's ([5]) solution to the famous "peak point problem".

The first author of the present paper has made use of Swiss cheese constructions to produce a variety of examples of plane sets $X$ such that the uniform algebra $R(X)$ has interesting, specified properties. In [8] he used McKissick's example, together with a system of Cole extensions, to construct a non-trivial, strongly regular uniform algebra (see that paper for the definition). In [9] the first author used a Swiss cheese construction to obtain a compact plane set $X$ such that $R(X)$ has no non-trivial Jensen measures (see that paper for the definition) but is not normal. In [10] the same author used a Swiss cheese construction to produce a counterexample to the conjecture (of Morris, in [17]) that a uniform algebra with no non-zero, bounded point derivations would have to be weakly amenable (see [6, Section 2.8]). In [12] the second author of the present paper showed that the uniform algebra produced could, in addition, be normal, by using a Swiss-cheese-like method of removing discs from a compact 2-cell; more details may be found in [13].

1.3.1. An issue in the literature. Constructions using non-classical Swiss cheeses (including the non-classical Swiss cheese constructions listed above) often rely upon the following result, which appears on pages 28 and 29 of [3].

Proposition 1.11. Let $\mathbf{D}=\left(\Delta,\left\{D_{1}, \ldots, D_{n}\right\}\right)$ be a finite Swiss cheese. Then $\partial X_{\mathbf{D}}$ consists of a finite number of arcs of the circles $\partial \Delta$ and $\partial D_{i}$. If we orient the arcs in $\partial \Delta \cap \partial X_{\mathbf{D}}$ positively and those of each $\partial D_{i} \cap \partial X_{\mathbf{D}}$ 
negatively, this turns $\partial X_{\mathbf{D}}$ into a contour such that the following holds. If $D$ is an open neighbourhood of $X_{\mathbf{D}}$ and $f$ is analytic on $D$, then

1. $\int_{\partial X_{\mathbf{D}}} f(z) d z=0$;

2. $f(\zeta)=\frac{1}{2 \pi i} \int_{\partial X_{\mathbf{D}}} \frac{f(z)}{z-\zeta} d z\left(\zeta \in \operatorname{int}\left(X_{\mathbf{D}}\right)\right)$.

However, the proof of this given in 3 is a sketch, which appears somewhat difficult to make rigorous, and we are not aware of any other proof of the result in print. This being the case, it may be helpful to have other methods available that do not depend on this result. Theorem 2.1 of the current paper will provide an alternative means of proving the non-triviality of $R(X)$ for a large class of non-classical Swiss cheese sets $X$.

We shall also show that in many cases of Swiss cheeses $\mathbf{D}$ constructed so that $R\left(X_{\mathbf{D}}\right)$ has particular properties, we may assume that $\mathbf{D}$ is classical without losing the relevant properties. In the following subsection we show that this will mean that we have natural, essential uniform algebras with a variety of specified properties on a fixed compact space, namely the Sierpiński carpet.

1.4. The Sierpiński carpet. The Sierpiński carpet is a well known fractal, which has been widely studied in topology, the theory of dynamical systems and complex analysis (see [2]). It is defined as follows. We let $Q$ be the compact 2-cell (rectangle) with corners at $0,1, i$ and $1+i$ and, for $z \in \mathbb{C}$ and $l \in] 0, \infty[$, we define $U(z, l)$ to be the open 2-cell with corners at $z, z+l, z+l i$ and $z+l+l i$. The Sierpinski carpet, $S$, is the set

$$
S=Q \backslash \bigcup_{k \in \mathbb{N}, m, n \in\left\{0, \ldots 3^{k-1}\right\}} U\left(3^{-k}((3 m+1)+(3 n+1) i), 3^{-k}\right) .
$$

Figure 1 shows an approximation of the Sierpiński carpet.

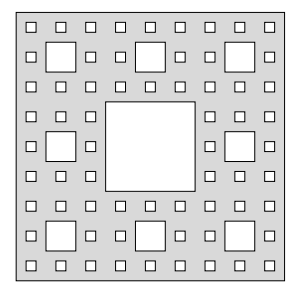

Fig. 1. The Sierpiński carpet

In this paper we consider how Swiss cheeses relate to plane homeomorphs of the Sierpiński carpet. Our first examples come as consequences of the following result of Whyburn ([24]), which may be found as [2, Theorem 7.2]. 
Proposition 1.12. Let $\Delta=\{z \in \mathbb{C}:|z| \leq 1\}$, let $\left(D_{i}\right)_{i \in \mathbb{N}}$ be a sequence of pairwise disjoint Jordan domains whose closures lie in the interior of $\Delta$, and let

$$
X:=\Delta \backslash \bigcup_{i \in \mathbb{N}} D_{i} .
$$

Then $X$ is homeomorphic to the Sierpiniski carpet if and only if $X$ has empty interior, $\partial D_{i} \cap \partial D_{j}=\emptyset$ for $i \neq j$, and $\operatorname{diam}\left(D_{i}\right) \rightarrow 0$ as $i \rightarrow \infty$.

This gives us the following corollary.

Corollary 1.13. Let $X$ be a classical Swiss cheese set with empty interior in $\mathbb{C}$. Then $X$ is homeomorphic to the Sierpinski carpet.

Thus, for any classical Swiss cheese set $X$ with empty interior we may consider $R(X)$ to be a uniform algebra on the Sierpiński carpet. Each of these algebras is natural and, by Theorem 1.8, each is essential. We now consider how a well known topological property of the Sierpiński carpet relates to uniform algebras.

Definition 1.14. Let $T$ be a non-empty topological space and $\mathcal{U} \in$ $\mathcal{P}(\mathcal{P}(T))$ be an open cover of $T$. We say an open cover $\mathcal{V}$ is a refinement of $\mathcal{U}$ if, for each $V \in \mathcal{V}$, there exists $U \in \mathcal{U}$ with $V \subseteq U$. We define the topological dimension of $T$ to be the smallest non-negative integer $n$ (if it exists) such that every open cover of $T$ has a refinement $\mathcal{V}$ such that each $x \in T$ is in at most $n+1$ elements of $\mathcal{V}$. If no such integer exists, then we say the toplogical dimension is infinite.

For subsets of $\mathbb{R}^{n}$ the following, which is [14, Theorem IV 3], holds.

Proposition 1.15. A subset $X$ of $\mathbb{R}^{n}$ has topological dimension strictly less than $n$ if and only if $X$ has empty interior in $\mathbb{R}^{n}$.

Definition 1.16. A compact plane set $X$ is a universal plane curve if it has topological dimension 1, and whenever $Y$ is a compact plane set with topological dimension less than or equal to 1 , then there is a subset $Y^{\prime}$ of $X$ which is homeomorphic to $Y$.

The following was proven by Sierpiński in [21] (see also [1, p. 433]).

Proposition 1.17. The Sierpinski carpet is a universal plane curve.

The remainder of this paper deals with a technique for finding classical Swiss cheese sets (and thus homeomorphs of the Sierpiński carpet) as subsets of plane sets that are built using Swiss cheeses, such as those discussed in our survey. 
2. Classicalisation of Swiss cheeses. For a Swiss cheese $\mathbf{D}=(\Delta, \mathcal{D})$, we define $\delta(\mathbf{D})=r(\Delta)-\sum_{D \in \mathcal{D}} r(D)$. Note that $\delta(\mathbf{D})>-\infty$ if and only if $\sum_{D \in \mathcal{D}} r(D)<\infty$. We shall prove the following theorem.

Theorem 2.1. For every Swiss cheese $\mathbf{D}$ with $\delta(\mathbf{D})>0$, there is a classical Swiss cheese $\mathbf{D}^{\prime}$ with $X_{\mathbf{D}^{\prime}} \subseteq X_{\mathbf{D}}$ and $\delta\left(\mathbf{D}^{\prime}\right) \geq \delta(\mathbf{D})$.

Now, most of the examples mentioned in the survey section of this paper allow us to make a free choice of $\Delta$, and to specify that $\sum_{D \in \mathcal{D}} r(D)$ be arbitrarily small. Hence, those plane sets may be taken to contain a classical Swiss cheese set as a subset. This is important because, if $X$ and $Y$ are compact plane sets with $Y \subseteq X$, then many properties of $R(X)$ are shared by $R(Y)$. We give some examples in the following proposition, which is elementary, is probably well known, and appears as [13, Lemma 2.1.1].

Proposition 2.2. Let $X$ and $Y$ be compact plane sets with $Y \subseteq X$. Then:

(i) if $R(X)$ is trivial then so is $R(Y)$;

(ii) if $R(X)$ does not have any non-zero bounded point derivations, then neither does $R(Y)$;

(iii) if $R(X)$ is normal, then so is $R(Y)$.

In order to prove Theorem 2.1 we shall need the following collection of facts. The proofs are elementary and may be found in [13].

Proposition 2.3.

(a) Let $\mathcal{F}$ be a non-empty, nested collection of open discs in $\mathbb{C}$ such that $\sup \{r(E): E \in \mathcal{F}\}<\infty$. Then $\bigcup \mathcal{F}$ is an open disc, $E$, and there is a nested increasing sequence $\left(D_{n}\right)_{n \in \mathbb{N}} \subseteq \mathcal{F}$ such that $\bigcup_{n \in \mathbb{N}} D_{n}=E$. Furthermore, if we order $\mathcal{F}$ by inclusion, then

$$
r(E)=\lim _{n \rightarrow \infty} r\left(D_{n}\right)=\lim _{D \in \mathcal{F}} r(D)=\sup _{D \in \mathcal{F}} r(D) .
$$

(b) Let $\mathcal{F}$ be a non-empty, nested collection of closed discs in $\mathbb{C}$. Then $\Delta:=\bigcap \mathcal{F}$ is a closed disc or a singleton and there is a nested decreasing sequence $\left(D_{n}\right)_{n \in \mathbb{N}} \subseteq \mathcal{F}$ such that $\bigcap_{n \in \mathbb{N}} D_{n}=\Delta$. Furthermore, if we order $\mathcal{F}$ by reverse inclusion, then

$$
r(\Delta)=\lim _{n \rightarrow \infty} r\left(D_{n}\right)=\lim _{D \in \mathcal{F}} r(D)=\inf _{D \in \mathcal{F}} r(D) .
$$

Definition 2.4. Let $\mathbf{D}=(\Delta, \mathcal{D})$ be a Swiss cheese. We define

$$
\widetilde{\mathbf{D}}=\mathcal{D} \cup\{\mathbb{C} \backslash \Delta\} \text {. }
$$

Now let $\mathbf{E}=(H, \mathcal{E})$ be a second Swiss cheese, and let $f: \widetilde{\mathbf{D}} \rightarrow \widetilde{\mathbf{E}}$. We define $\mathcal{G}(f)=f^{-1}(\{\mathbb{C} \backslash H\}) \cap \mathcal{D}$. We say that $f$ is an allocation map if the following hold: 
(A1) for each $U \in \widetilde{\mathbf{D}}, U \subseteq f(U)$;

(A2)

$$
\sum_{D \in \mathcal{G}(f)} r(D) \geq r(\Delta)-r(H)
$$

(A3) for each $E \in \mathcal{E}$,

$$
\sum_{D \in f^{-1}(E)} r(D) \geq r(E)
$$

If there is an allocation map from $\widetilde{\mathbf{D}}$ to $\widetilde{\mathbf{E}}$ we say that $\mathbf{E}$ is above $\mathbf{D}$.

Note that these axioms imply that $f$ is surjective. In particular, since there is no disc $D$ with $\mathbb{C} \backslash \Delta \subseteq D$ we have $f(\mathbb{C} \backslash \Delta)=\mathbb{C} \backslash H$.

Thus, if $\mathbf{E}$ is above $\mathbf{D}$, then (A1) implies that $H \subseteq \Delta$. The following properties of allocation maps are elementary consequences of the definition. Full details of the proofs may be found in [13].

Proposition 2.5.

(i) Let $\mathbf{D}_{1}=\left(\Delta_{1}, \mathcal{D}_{1}\right), \mathbf{D}_{2}=\left(\Delta_{2}, \mathcal{D}_{2}\right)$ and $\mathbf{D}_{3}=\left(\Delta_{3}, \mathcal{D}_{3}\right)$ be Swiss cheeses and let

$$
f: \widetilde{\mathbf{D}}_{1} \rightarrow \widetilde{\mathbf{D}}_{2}, \quad g: \widetilde{\mathbf{D}}_{2} \rightarrow \widetilde{\mathbf{D}}_{3}
$$

be allocation maps. Then $g \circ f$ is an allocation map from $\widetilde{\mathbf{D}}_{1}$ to $\widetilde{\mathbf{D}}_{3}$.

(ii) Let $\mathbf{D}=(\Delta, \mathcal{D})$ be a Swiss cheese. Then the identity map from $\widetilde{\mathbf{D}}$ to itself is an allocation map. Suppose further that $\sum_{D \in \mathcal{D}} r(D)<\infty$. Then the identity map is the unique allocation map from $\widetilde{\mathbf{D}}$ to itself.

(iii) Suppose that $\mathbf{D}=(\Delta, \mathcal{D})$ and $\mathbf{E}=(H, \mathcal{E})$ are Swiss cheeses such that $\mathbf{E}$ is above $\mathbf{D}$. Then $X_{\mathbf{E}} \subseteq X_{\mathbf{D}}$.

(iv) Let $\mathbf{D}=(\Delta, \mathcal{D})$ and $\mathbf{E}=(H, \mathcal{E})$ be Swiss cheeses such that $\mathbf{E}$ is above $\mathbf{D}$. Then

$$
\delta(\mathbf{E}) \geq \delta(\mathbf{D}) .
$$

We note that parts (i) and (ii) of the preceding proposition show that taking Swiss cheeses as objects and allocation maps as morphisms gives a (small) category. Thus, we may consider subcategories such as the category of Swiss cheeses, D, such that $\delta(\mathbf{D})>0$ and allocation maps. Now fix a Swiss cheese $\mathbf{D}$, and let $\mathcal{S}(\mathbf{D})$ be the collection of all pairs $(\mathbf{E}, f)$ such that $\mathbf{E}$ is a Swiss cheese and $f: \widetilde{\mathbf{D}} \rightarrow \widetilde{\mathbf{E}}$ is an allocation map. Note that, for all $(\mathbf{E}, f) \in \mathcal{S}(D), \mathbf{E}$ is above $\mathbf{D}$. We define a binary relation, $\geq$, on $\mathcal{S}(\mathbf{D})$ by saying $\left(\mathbf{E}^{\prime}, f^{\prime}\right) \geq(\mathbf{E}, f)$ if there is an allocation map $g: \widetilde{\mathbf{E}} \rightarrow \widetilde{\mathbf{E}^{\prime}}$ such that $g \circ f=f^{\prime}$. Note that, since $f$ is onto, any such $g$ is unique.

Lemma 2.6. Let $\mathbf{D}$ be a Swiss cheese such that $\delta(\mathbf{D})>-\infty$. Then the binary relation $\geq$ defined above is a partial order on $\mathcal{S}(\mathbf{D})$. 
Proof. First, we show that $\geq$ is reflexive. Let $(\mathbf{E}, f) \in \mathcal{S}(\mathbf{D})$. By Proposition 2.5 the identity map id : $\widetilde{\mathbf{E}} \rightarrow \widetilde{\mathbf{E}}$ is an allocation map. Clearly, id $\circ f=f$ and so $(\mathbf{E}, f) \geq(\mathbf{E}, f)$.

Now, we show that $\geq$ is transitive. Let $\left(\mathbf{E}_{1}, f_{1}\right),\left(\mathbf{E}_{2}, f_{2}\right),\left(\mathbf{E}_{3}, f_{3}\right) \in \mathcal{S}(\mathbf{D})$ be such that $\left(\mathbf{E}_{2}, f_{2}\right) \geq\left(\mathbf{E}_{1}, f_{1}\right)$ and $\left(\mathbf{E}_{3}, f_{3}\right) \geq\left(\mathbf{E}_{2}, f_{2}\right)$. Then there are allocation maps, $g_{1,2}: \widetilde{\mathbf{E}}_{1} \rightarrow \widetilde{\mathbf{E}}_{2}$ such that $g_{1,2} \circ f_{1}=f_{2}$, and $g_{2,3}: \widetilde{\mathbf{E}}_{2} \rightarrow \widetilde{\mathbf{E}}_{3}$ such that $g_{2,3} \circ f_{2}=f_{3}$. Set $g_{1,3}=g_{2,3} \circ g_{1,2}$. Then, by part (i) of Proposition 2.5. $g_{1,3}$ is an allocation map from $\widetilde{\mathbf{E}}_{1}$ to $\widetilde{\mathbf{E}}_{3}$. Also,

$$
g_{1,3} \circ f_{1}=\left(g_{2,3} \circ g_{1,2}\right) \circ f_{1}=g_{2,3} \circ\left(g_{1,2} \circ f_{1}\right)=g_{2,3} \circ f_{2}=f_{3},
$$

and so $\left(\mathbf{E}_{3}, f_{3}\right) \geq\left(\mathbf{E}_{1}, f_{1}\right)$.

Finally, we show that $\geq$ is antisymmetric. Let $\left(\mathbf{E}_{1}, f_{1}\right),\left(\mathbf{E}_{2}, f_{2}\right) \in \mathcal{S}(\mathbf{D})$ be such that $\left(\mathbf{E}_{2}, f_{2}\right) \geq\left(\mathbf{E}_{1}, f_{1}\right)$ and $\left(\mathbf{E}_{1}, f_{1}\right) \geq\left(\mathbf{E}_{2}, f_{2}\right)$. Then there are allocation maps, $g_{1,2}: \widetilde{\mathbf{E}}_{1} \rightarrow \widetilde{\mathbf{E}}_{2}$ such that $g_{1,2} \circ f_{1}=f_{2}$, and $g_{2,1}: \widetilde{\mathbf{E}}_{2} \rightarrow \widetilde{\mathbf{E}}_{1}$ such that $g_{2,1} \circ f_{2}=f_{1}$. Set

$$
g=g_{2,1} \circ g_{1,2}: \widetilde{\mathbf{E}}_{1} \rightarrow \widetilde{\mathbf{E}}_{1} .
$$

Then, by part (i) of Proposition 2.5, $g$ is an allocation map. Since $\mathbf{E}$ is above $D, \delta\left(\mathbf{E}_{1}\right)>-\infty$ and so, by part (ii) of Proposition 2.5, $g$ is the identity map on $\widetilde{\mathbf{E}}_{1}$. Now, let $U \in \widetilde{\mathbf{E}}_{1}$. It follows easily that

$$
U \subseteq g_{1,2}(U) \subseteq g_{2,1}\left(g_{1,2}(U)\right)=g(U)=U,
$$

so $g_{1,2}(U)=U$. Similarly, if $U \in \widetilde{\mathbf{E}}_{2}$, then $g_{2,1}(U)=U$. Thus $\left(\mathbf{E}_{1}, f_{1}\right)=$ $\left(\mathbf{E}_{2}, f_{2}\right)$.

Lemma 2.7. Let $\mathbf{D}$ be a Swiss cheese such that $\delta(\mathbf{D})>0$, and let $\mathcal{C}$ be a chain in $(\mathcal{S}(\mathbf{D}), \geq)$. Then $\mathcal{C}$ has an upper bound in $(\mathcal{S}(\mathbf{D}), \geq)$.

Proof. For $i \in \mathcal{C}$ we write $i=\left(\mathbf{E}_{i}, f_{i}\right)$ and $\mathbf{E}_{i}=\left(H_{i}, \mathcal{E}_{i}\right)$, and for $j \in \mathcal{C}$ with $j \geq i$, we let

$$
g_{i, j}: \widetilde{\mathbf{E}}_{i} \rightarrow \widetilde{\mathbf{E}}_{j}
$$

be the unique (as discussed above) allocation map such that $g_{i, j} \circ f_{i}=f_{j}$. From uniqueness, it follows easily that

$$
g_{i, k}=g_{j, k} \circ g_{i, j} \quad(i \leq j \leq k \in \mathcal{C}) .
$$

Note that $\left\{H_{i}: i \in \mathcal{C}\right\}$ is a nested decreasing collection of closed discs and, for each $D \in \mathcal{D},\left\{f_{i}(D): i \in \mathcal{C}\right\}$ is a nested increasing collection of open plane sets.

By part (iv) of Proposition 2.5, we have

$$
r\left(H_{i}\right) \geq \delta\left(\mathbf{E}_{i}\right) \geq \delta(\mathbf{D})>0 .
$$

Let $H=\bigcap_{i \in \mathcal{C}} H_{i}$. By part (b) of Proposition 2.3. $H$ is a compact disc or singleton with

$$
r(H)=\lim _{i \in \mathcal{C}} r\left(H_{i}\right)
$$


By (2), $r(H)>0$, so $H$ is a compact disc. Now we define a map as follows:

$$
f: \widetilde{\mathbf{D}} \rightarrow \mathcal{P}(\mathbb{C}), \quad U \mapsto \bigcup_{i \in \mathcal{C}} f_{i}(U) .
$$

First, we note that

$$
f(\mathbb{C} \backslash \Delta)=\bigcup_{i \in \mathcal{C}}\left(\mathbb{C} \backslash H_{i}\right)=\mathbb{C} \backslash \bigcap_{i \in \mathcal{C}} H_{i}=\mathbb{C} \backslash H .
$$

Note also that, if $D \in \mathcal{D}$, then exactly one of the following two cases holds:

(i) There exists $i \in \mathcal{C}$ such that $f_{i}(D)=\mathbb{C} \backslash H_{i}$. In this case, for $j \geq i$, we have $f_{j}(D)=g_{i, j}\left(\mathbb{C} \backslash H_{i}\right)=\mathbb{C} \backslash H_{j}$, since $f_{j}=g_{i, j} \circ f_{i}$. Thus $f(D)=\mathbb{C} \backslash H$.

(ii) For each $i \in \mathcal{C}, f_{i}(D) \in \mathcal{E}_{i}$. In this case, $\left\{f_{i}(D): i \in \mathcal{C}\right\}$ is a collection of open discs with $f_{i}(D) \subseteq f_{j}(D)$ if $i \leq j$. Also, for each $i \in \mathcal{C}$, we have (since $f_{i}$ satisfies (A3))

$$
r\left(f_{i}(D)\right) \leq \sum_{D \in f_{i}^{-1}\left(f_{i}(D)\right)} r(D) \leq \sum_{D \in \mathcal{D}} r(D)<\infty .
$$

Thus, by part (a) of Proposition 2.3, $f(D)=\bigcup_{i \in \mathcal{C}} f_{i}(D)$ is an open disc with $r(f(D))=\lim _{i \in \mathcal{C}} r\left(f_{i}(D)\right)$.

Hence,

$$
\mathcal{E}:=\{f(U): U \in \mathcal{D}\} \backslash\{\mathbb{C} \backslash H\}
$$

is a collection of open discs.

Set $\mathbf{E}=(H, \mathcal{E})$. By the above, $\mathbf{E}$ is a Swiss cheese. By definition, $f(\widetilde{\mathbf{D}})=\widetilde{\mathbf{E}}$. We claim that $f$ (considered as map into $\widetilde{\mathbf{E}}$ ) is an allocation map. That $f$ satisfies (A1) is trivial.

To show that $f$ satisfies (A2), note that, by the argument for case (i) above,

$$
\bigcup_{i \in \mathcal{C}}\left(f_{i}^{-1}\left(\mathbb{C} \backslash H_{i}\right)\right) \subseteq f^{-1}(\mathbb{C} \backslash H)
$$

i.e.

$$
\mathcal{G}_{i} \subseteq \mathcal{G}(f) \quad(i \in \mathcal{C}) .
$$

Thus, since $r(H)=\lim _{i \in \mathcal{C}} r\left(H_{i}\right)$, and each $f_{i}$ satisfies (A2), we have

$$
\begin{aligned}
r(H) & =\liminf _{i \in \mathcal{C}} r\left(H_{i}\right) \geq \liminf _{i \in \mathcal{C}}\left(r(\Delta)-\sum_{D \in \mathcal{G}\left(f_{i}\right)} r(D)\right) \\
& \geq r(\Delta)-\sum_{D \in \mathcal{G}(f)} r(D) .
\end{aligned}
$$

Hence, $f$ satisfies (A2). 
To show that $f$ satisfies (A3), let $E \in \mathcal{E}$, and let $U \in \mathcal{D}$ be such that $f(U)=E$. Let $i, j \in \mathcal{C}$ with $j \geq i$, and let $D \in \mathcal{D}$ be such that $f_{i}(U)=f_{i}(D)$. Then, since $f_{j}=g_{i, j} \circ f_{i}$, we have $f_{j}(D)=f_{j}(U)$, and so $f(D)=f(U)=E$. Thus,

$$
\bigcup_{i \in \mathcal{C}} f_{i}^{-1}\left(f_{i}(U)\right) \subseteq f^{-1}(f(U))=f^{-1}(E) .
$$

Since $r(E)=\lim _{i \in \mathcal{C}} r\left(f_{i}(U)\right)$, and each $f_{i}$ satisfies (A3), we have

$$
\begin{aligned}
r(E) & =\limsup _{i \in \mathcal{C}} r\left(f_{i}(U)\right) \leq \limsup _{i \in \mathcal{C}}\left(\sum_{D \in f_{i}^{-1}\left(f_{i}(U)\right)} r(D)\right) \\
& \leq \sum_{D \in f^{-1}(E)} r(D) .
\end{aligned}
$$

Thus $f$ satisfies (A3), and so is an allocation map.

We claim that $(\mathbf{E}, f)$ is the upper bound we require. To see this, let $i=\left(\mathbf{E}_{i}, f_{i}\right) \in \mathcal{C}$, define $I_{i}=\{j \in \mathcal{C}: j \geq i\}$, and take $U \in \widetilde{\mathbf{E}}_{i}$. Then, there exists $V \in \widetilde{\mathbf{D}}$ such that $U=f_{i}(V)$. Now let $j \in \mathcal{C}$ with $j \geq i$. Then $g_{i, j}(U)=f_{j}(V)$. Thus we have

$$
\bigcup_{j \in I_{i}} g_{i, j}(U)=\bigcup_{j \in \mathcal{C}} f_{j}(V)=f(V) \in \widetilde{\mathbf{E}} .
$$

Hence, we can define a map

$$
g_{i}: \widetilde{\mathbf{E}}_{i} \rightarrow \widetilde{\mathbf{E}}, \quad U \mapsto \bigcup_{j \in I_{i}} g_{i, j}(U),
$$

and we have $g_{i} \circ f_{i}=f$.

It remains to show that $g_{i}$ is an allocation map. To see this, note that it follows from the equation (1) that $I_{i}$ is a chain in $\mathcal{S}\left(\mathbf{E}_{i}\right)$, and so the proof that $f$ is an allocation map also shows that $g_{i}$ is. The result follows.

Proof of Theorem 2.1. By Proposition 2.5 (part (ii)) and Lemmas 2.6 and 2.7. $(\mathcal{S}(\mathbf{D}), \geq)$ is a non-empty, partially ordered set such that every chain has an upper bound. Hence, we may apply Zorn's lemma to obtain a maximal element $(\mathbf{E}, f)$ of $(\mathcal{S}(\mathbf{D}), \geq)$. By part (iii) of Proposition 2.5, we have $X_{\mathbf{E}} \subseteq X_{\mathbf{D}}$. Since $\mathbf{E}$ is above $\mathbf{D}, \delta(\mathbf{E}) \geq \delta(\mathbf{D})>0$.

It remains to show that $\mathbf{E}$ is a classical Swiss cheese. Towards a contradiction, we assume otherwise. Then we must have at least one of the following cases.

CASE 1: There exist $E, E^{\prime} \in \mathcal{E}$ such that $\bar{E} \cap \bar{E}^{\prime} \neq \emptyset$. In this case there exists an open disc $E^{\prime \prime}$ with $E \cup E^{\prime} \subseteq E^{\prime \prime}$ and $r\left(E^{\prime \prime}\right) \leq r(E)+r\left(E^{\prime}\right)$, as in Figure 2. Let $\mathcal{E}^{\prime}=\left(\mathcal{E} \backslash\left\{E, E^{\prime}\right\}\right) \cup\left\{E^{\prime \prime}\right\}$ and $\mathbf{E}^{\prime}=\left(H, \mathcal{E}^{\prime}\right)$ and define 


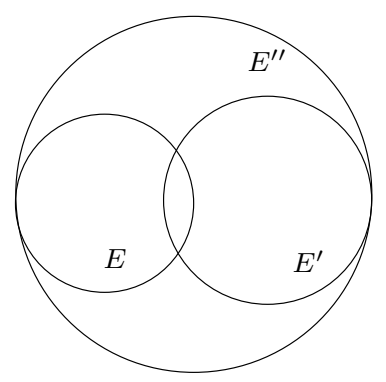

Fig. 2. $E, E^{\prime}$ and $E^{\prime \prime}$

$g: \widetilde{\mathbf{E}} \rightarrow \widetilde{\mathbf{E}^{\prime}}$ by

$$
g(U)= \begin{cases}E^{\prime \prime} & \text { if } U \in\left\{E, E^{\prime}\right\} \\ U & \text { otherwise. }\end{cases}
$$

Then it is easy to check that $g$ is an allocation map. By part (i) of Proposition 2.5. $g \circ f$ is an allocation map, and so $\left(\mathbf{E}^{\prime}, f \circ g\right) \in \mathcal{S}(\mathbf{D})$ with $\left(\mathbf{E}^{\prime}, f \circ g\right)>$ $(\mathbf{E}, f)$.

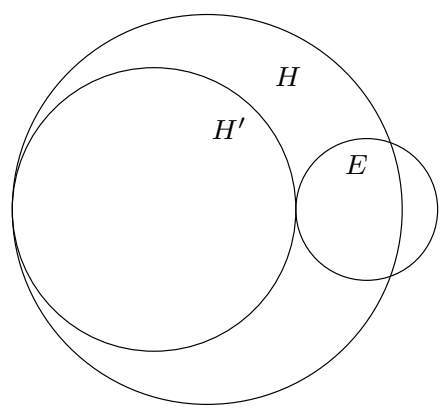

Fig. 3. $E, H$ and $H^{\prime}$

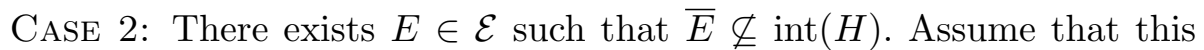
case holds and Case 1 does not. By the condition on the sum of the radii, $\operatorname{int}(H) \nsubseteq \bar{E}$. Then there exists a compact disc $H^{\prime}$ such that $D \subseteq \mathbb{C} \backslash H^{\prime}$ and $r\left(H^{\prime}\right) \geq r(H)-r(E)$ as in Figure 3. Let $\mathcal{E}^{\prime}=\mathcal{E} \backslash\{E\}$ and $\mathbf{E}^{\prime}=\left(H^{\prime}, \mathcal{E}^{\prime}\right)$ and define $g: \widetilde{\mathbf{E}} \rightarrow \widetilde{\mathbf{E}^{\prime}}$ by

$$
g(U)= \begin{cases}\mathbb{C} \backslash H^{\prime} & \text { if } U \in\{E, \mathbb{C} \backslash H\} \\ U & \text { otherwise. }\end{cases}
$$

Then it is easy to check that $g$ is an allocation map. By part (i) of Proposition 2.5. $g \circ f$ is an allocation map, and so $\left(\mathbf{E}^{\prime}, f \circ g\right) \in \mathcal{S}(\mathbf{D})$ with $\left(\mathbf{E}^{\prime}, f \circ g\right)>$ $(\mathbf{E}, f)$. 
In either case we have a contradiction to the maximality of $(\mathbf{E}, f)$. The result follows.

We are grateful to Prof. J. K. Langley for pointing out to us that the method we use to combine discs in Case 1 has previously appeared in the literature in the setting of finite unions of open discs. Zhang implicitly uses this method on page 50 of [25].

Theorem 2.1 has the following purely topological corollary.

Corollary 2.8. Let $\mathbf{D}$ be a Swiss cheese such that $\delta(\mathcal{D})>0$ and $X_{\mathbf{D}}$ has empty interior in $\mathbb{C}$. Then $X_{\mathbf{D}}$ is a universal plane curve.

Proof. By Theorem 2.1 there is a classical Swiss cheese set $Y$ with $Y \subseteq X_{\mathbf{D}}$. By Corollary 1.13, $Y$ is homeomorphic to the Sierpiński carpet $S$. Let $E$ be a compact plane set with topological dimension less than or equal to 1 . Then by Proposition 1.17 there is a plane set $E^{\prime}$ homeomorphic to $E$ with $E^{\prime} \subseteq Y \subseteq X_{\mathbf{D}}$.

Note, in particular, that for any two such Swiss cheese sets, each may be continuously embedded in the other.

We are now able to use known examples of non-classical Swiss cheeses $X$ such that $R(X)$ has particular properties to construct new examples using classical Swiss cheeses (in particular, to produce examples of essential uniform algebras on the Sierpiński carpet). We give the following example.

EXAmple 2.9. There is a classical Swiss cheese set $X$ such that $R(X)$ is normal.

Proof. By Proposition 1.10, there is a Swiss cheese $\mathbf{D}=(\Delta, \mathcal{D})$ such that

$$
\delta(\mathbf{D})>0
$$

and $R\left(X_{\mathbf{D}}\right)$ is normal. By Theorem 2.1, there is a classical Swiss cheese set $X$ with $X \subseteq X_{\mathbf{D}}$. By Proposition 2.2, $R(X)$ is normal.

We note that, by Propositions 1.9 and 2.2 , we could in addition insist that $R(X)$ have no non-zero bounded point derivations.

We do not yet know whether the techniques in this paper can be adapted so that they preserve the existence of point derivations, or of other derivations into the dual of $R(X)$. Proofs of the existence of such derivations often use Proposition 1.11 (see, for example, [10]). If one wishes to avoid using that result, other "work-arounds" can typically be found (see, for example, Theorem 3.3.8 of [13]).

3. Open questions. We finish with some open questions.

Question 3.1. Let $X$ be a compact plane set such that $R(X) \neq C(X)$. Does it follow that $X$ has a subset $S$ homeomorphic to the Sierpinski carpet? 
Question 3.2. Let $X$ be a compact plane set such that $R(X) \neq C(X)$. Does it follow that $X$ has a subset $S$ homeomorphic to the Sierpinski carpet such that $R(S) \neq C(X)$ ?

Question 3.3. Let $X$ be a plane set such that $R(X) \neq C(X)$. Does it follow that $X$ has a subset which is a classical Swiss cheese set?

QUESTION 3.4. Let $X$ be a compact plane set such that there exists a non-trivial, natural uniform algebra on $X$. Does it follow that $X$ has a subset homeomorphic to the Sierpinski carpet?

QUESTION 3.5. Let $X$ be a compact metric space such that there exists a non-trivial, natural uniform algebra on $X$. Does it follow that $X$ has a subset homeomorphic to the Sierpinski carpet?

We note that a positive answer to Question 3.4 would imply a negative answer to the following - a famous problem due to Gel'fand.

QUESTION 3.6. Is there a non-trivial, natural uniform algebra on the interval $[0,1]$ ?

Acknowledgments. M. J. Heath is supported by post-doctoral grant SFRH/BPD/40762/2007 from FCT (Portugal) and completed most of this research while supported by a PhD grant from the EPSRC (UK). This paper contains work from his $\mathrm{PhD}$ thesis [13].

\section{References}

[1] L. M. Blumenthal and K. Menger, Studies in Geometry, W. H. Freeman, San Francisco, CA, 1970.

[2] M. Bonk, Quasiconformal geometry of fractals, in: Internat. Congress of Mathematicians (Madrid, 2006), Vol. II, Eur. Math. Soc., Zürich, 2006, 1349-1373.

[3] F. F. Bonsall and J. Duncan, Complete Normed Algebras, Ergeb. Math. Grenzgeb. 80, Springer, New York, 1973.

[4] A. Browder, Introduction to Function Algebras, W. A. Benjamin, New York, 1969.

[5] B. J. Cole, One point parts and the peak point conjecture, Ph.D. thesis, Yale Univ., 1968.

[6] H. G. Dales, Banach Algebras and Automatic Continuity, London Math. Soc. Monogr. (N.S.) 24, Clarendon Press, New York, 2000.

[7] T. Dawson, A survey of algebraic extensions of commutative, unital normed algebras, in: Function Spaces (Edwardsville, IL, 2002), Contemp. Math. 328, Amer. Math. Soc., Providence, RI, 2003, 157-170.

[8] J. F. Feinstein, A nontrivial, strongly regular uniform algebra, J. London Math. Soc. (2) 45 (1992), 288-300.

[9] -, Trivial Jensen measures without regularity, Studia Math. 148 (2001), 67-74.

[10] -, A counterexample to a conjecture of S. E. Morris, Proc. Amer. Math. Soc. 132 (2004), 2389-2397.

[11] T. W. Gamelin, Uniform Algebras, Prentice-Hall, Englewood Cliffs, NJ, 1969. 
[12] M. J. Heath, A note on a construction of J. F. Feinstein, Studia Math. 169 (2005), 63-70.

[13] —, Bounded derivations from Banach algebras, Ph.D. thesis, Univ. of Nottingham, 2008.

[14] W. Hurewicz and H. Wallman, Dimension Theory, Princeton Math. Ser. 4, Princeton Univ. Press, Princeton, NJ, 1941.

[15] T. W. Körner, A cheaper Swiss cheese, Studia Math. 83 (1986), 33-36.

[16] R. McKissick, A nontrivial normal sup norm algebra, Bull. Amer. Math. Soc. 69 (1963), 391-395.

[17] S. E. Morris, Bounded derivations from uniform algebras, Ph.D. thesis, Univ. of Cambridge, 1993.

[18] A. G. O'Farrell, A regular uniform algebra with a continuous point derivation of infinite order, Bull. London Math. Soc. 11 (1979), 41-44.

[19] A. Roth, Approximationseigenschaften und Strahlengrenzwerte meromorpher und ganzer Funktionen, Comment. Math. Helv. 11 (1938), 77-125.

[20] W. Rudin, Real and Complex Analysis, McGraw-Hill, Singapore, 1987.

[21] W. Sierpiński, Sur une courbe cantorienne qui contient une image biunivoque et continue de toute courbe donnée, in: Oeuvres choisies, tome II, PWN, Warszawa, 1975, 107-119 (originally published in Russian: Mat. Sb. 30 (1916), 267-287).

[22] L. A. Steen, On uniform approximation by rational functions, Proc. Amer. Math. Soc. 17 (1966), 1007-1011.

[23] J. Wermer, Bounded point derivations on certain Banach algebras, J. Funct. Anal. 1 (1967), 28-36.

[24] G. T. Whyburn, Topological characterization of the Sierpinski curve, Fund. Math. 45 (1958), 320-324.

[25] G. H. Zhang, Theory of Entire and Meromorphic Functions. Deficient and Asymptotic Values and Singular Directions, Transl. Math. Monogr. 122, Amer. Math. Soc., Providence, RI, 1993.

J. F. Feinstein

School of Mathematical Sciences

University of Nottingham

University Park

Nottingham, NG7 2RD, UK

E-mail: Joel.Feinstein@nottingham.ac.uk
M. J. Heath

Departamento de Matemática Instituto Superior Técnico Av. Rovisco Pais 1049-001 Lisboa, Portugal E-mail: mheath@math.ist.utl.pt 